\title{
Periprocedural Variability of Platelet Original Functions in Carotid Artery Stenting: An Analysis Using VerifyNow
}

Masataka Yoshimura, ${ }^{1,2}$ Kazutaka Sumita, ${ }^{3}$ Shoko Fujii, ${ }^{3}$ Kazunori Miki, ${ }^{3}$ Yuki Aizawa, ${ }^{3}$ Kyohei Fujita, ${ }^{3}$ Shinji Yamamoto, ${ }^{2}$ Shigeru Nemoto, ${ }^{3}$ and Taketoshi Maehara ${ }^{1}$

Objective: The assessment of platelet functions is necessary to prevent both thromboembolic and hemorrhagic complications under dual antiplatelet therapy (DAPT). Using the VerifyNow (Accumetrics, Inc., San Diego, CA, USA) assay, this study aimed to reveal time-dependent changes in platelet functions after carotid artery stenting (CAS).

Methods: We enrolled retrospectively 43 patients who underwent CAS under DAPT. Aspirin reaction unit (ARU) and $\mathrm{P} 2 \mathrm{Y} 12$ reaction unit (PRU) values were determined on the day before and on days 1,3 , and 7 after the procedure. Multiple comparison tests (MCTs) were performed among ARU and PRU measurement points, and the proportions of hypo- and hyper-responses were compared.

Results: The median ARU values were 408 (interquartile range: 392-497) before CAS and 418 (405-470) on day 1, 405 (393.0-460.5) on day 3, and 402 (388.5-477.5) on day 7 (not significant in MCTs). The percentages of hypo-responses were $16.3 \%, 7.0 \%, 2.3 \%$, and $7.0 \%$, respectively $(p=0.11)$. The significantly different median PRU values were 173 (116.5-209.5), 233 (166.5-273.5), 139 (70.5-205.5), and 51 (9.0-79.5), respectively. The median PRU was before the procedure within the therapeutic range but exceeded the upper cutoff on day 1 and was below the lower cutoff on day 7 . The percentages of hypo-responses were $14.0 \%, 51.2 \%, 18.6 \%$, and $11.6 \%$, respectively $(p<0.001)$ and the percentages of hyper-responses were $9.3 \%, 2.3 \%, 23.3 \%$, and $62.8 \%$, respectively $(p<0.001)$.

Conclusion: In the periprocedural CAS period, ARU values were stable, but PRU values showed time-dependent changes. PRU values were above the therapeutic range the day after CAS but decreased below this range on day 7 .

Keywords $>$ carotid artery stenting, platelet function, VerifyNow

\section{Introduction}

Carotid artery stenosis is a major cause of ischemic stroke, and carotid endarterectomy is an established treatment to prevent stroke. In recent years, carotid artery stenting

$\overline{{ }^{1} \text { Department of Neurosurgery, Tokyo Medical and Dental }}$ University, Tokyo, Japan

${ }^{2}$ Department of Neurosurgery, Tsuchiura Kyodo General Hospital, Tsuchiura, Ibaraki, Japan

${ }^{3}$ Department of Endovascular Surgery, Tokyo Medical and Dental University, Tokyo, Japan

Received: September 3, 2020; Accepted: October 31, 2020 Corresponding author: Masataka Yoshimura. Department of Neurosurgery, Tokyo Medical and Dental University, 1-5-45, Yushima, Bunkyo-ku, Tokyo 113-8519, Japan Email:mstkktsm@yahoo.co.jp

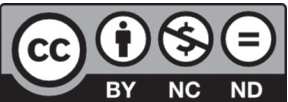

This work is licensed under a Creative Commons Attribution-NonCommercial-

NoDerivatives International License.

(C)2021 The Japanese Society for Neuroendovascular Therapy
(CAS) has provided a less invasive alternative, especially in endarterectomy high-risk patients. ${ }^{1)}$ Although emboli protection devices are effective in CAS, the procedure requires attention to thromboembolic complications such as in-stent thrombosis and acute stent occlusion in the perioperative period. ${ }^{2)}$ Therefore, the administration of antiplatelet drugs is important in CAS during the periprocedural period to prevent thromboembolic complications. ${ }^{3)}$ Dual antiplatelet therapy (DAPT) with clopidogrel plus low-dose aspirin is recommended because each drug inhibits the platelet function via a different pathway. When using antiplatelet drugs, we should pay attention to insufficient antiplatelet effects causing thromboembolic complications during endovascular treatment. This effect is termed "hypo-response," also known as "high on-treatment platelet reactivity" or "resistance." Besides, DAPT increases the risk of life-threatening hemorrhagic complications. ${ }^{12)}$ Therefore, we need to manage DAPT in the optimum range to prevent not only thromboembolic but also hemorrhagic complications. 
Conventional methods for assessing platelet function are complicated and difficult to standardize. The VerifyNow (Accumetrics, Inc., San Diego, CA, USA) assay analyses platelet functions quickly and easily. ${ }^{4)}$ Many studies using VerifyNow have provided useful evidence about hypo-response to aspirin or clopidogrel, ${ }^{4-11,13-17)}$ as well as hyper-response to clopidogrel. ${ }^{4,13,18)}$ Recently, dynamic variability in response to clopidogrel after endovascular treatment has been reported ${ }^{10,18-23)}$; however, individual changes in platelet functions in the short period under DAPT have not been described yet. Using the VerifyNow assay, this study aimed to reveal how and why platelet functions in CAS patients change in the periprocedural period.

\section{Materials and Methods}

\section{Patient population}

This study was approved by the ethical review board of each study institution and was conducted in accordance with the Declaration of Helsinki. All patients gave written informed consent for their participation in the study. Patients who underwent CAS for carotid artery stenosis at these institutes between March 2013 and August 2018 were enrolled in this study. Patients who underwent CAS for carotid artery dissection or restenosis after CAS were excluded. Thus, we obtained chronological VerifyNow data from 51 patients. Another exclusion criterion was insufficient antiplatelet pretreatment before the procedure. Sufficient antiplatelet pretreatment was defined as more than $7 \mathrm{~d}$ of $100 \mathrm{mg} / \mathrm{d}$ aspirin and $14 \mathrm{~d}$ of $75 \mathrm{mg} / \mathrm{d}$ clopidogrel. Eight patients were excluded based on this criterion. There was no control group in this study.

\section{Methods}

Using VerifyNow, we determined the parameters aspirin reaction unit (ARU) and $\mathrm{P} 2 \mathrm{Y} 12$ reaction unit (PRU) at day 1 before and days 1, 3, and 7 after the CAS procedure. Blood samples of the patients were collected into $2.0 \mathrm{~mL}$ $3.2 \%$ sodium citrate tubes from the median cubital vein or an arterial line inserted in the radial artery. All VerifyNow measurements were performed within 2 hours of sampling. Using the commonly accepted cutoff values of 550 ARU, aspirin responses above 550 were defined as hypo-response. For clopidogrel, PRU results above 230 were defined as hypo-response, ${ }^{5)}$ in the range of 70-230 as normal response, and below 70 as hyper-response. ${ }^{13)}$

To determine factors affecting the initial state of response to DAPT and changes in platelet functions, the following data were recorded: age, sex, presence of hypertension, diabetes, hyperlipidemia, coronary artery disease, and current smoking at the time of recording, administration of statins, proton pump inhibitors, cilostazol, and oral anti-coagulants, preoperative laboratory values including serum albumin, hemoglobin, and platelets, symptomatic or asymptomatic lesion, and percentage of stenosis using the North American Symptomatic Carotid Endarterectomy Trial (NASCET) method. ${ }^{24)}$ Appearance of diffusion-weighted image (DWI)positive lesions, symptomatic ischemic complications and hemorrhagic complications were also recorded and the relation between responsiveness at each measurement point and the occurrence of symptomatic complications and the appearance of DWI-positive lesions were analyzed.

\section{CAS procedure}

Almost all procedures were performed under general anesthesia apart from 1 case in which general anesthesia was undesirable. An arterial line was placed into the radial artery for invasive arterial pressure monitoring in all cases. A guiding catheter was inserted from the right or left femoral artery after systemic heparinization with a target activated clotting time of 250-300 s. If we experienced difficulties with this femoral approach, we chose the radial approach. Emboli protection devices were selected by the operator but were used in all cases. We used the Carotid Wallstent (Boston Scientific, Natick, MA, USA) which has a closed-cell design except in cases with highly curved lesions, where we employed stents with open-cell design (Precise; Cordis, Miami, FL, USA and Protégé RX; Medtronic, Minneapolis, MN, USA). Predilation of the stenotic lesion by a percutaneous trans-arterial balloon was only performed if the stent delivery system failed to pass through the lesion. After the procedure, systemic heparinization was discontinued, and DAPT was maintained for at least 2 months. We did not add antiplatelet dosages according to the test results unless there was an ischemic complication after the stenting procedure.

\section{Statistical analysis}

Continuous variables were analyzed using the MannWhitney U test and categorical variables using Fisher's exact test to compare between two groups. For multiple comparisons among either ARU or PRU values at different time points, the Wilcoxon signed-rank test adjusted by Holm's method was used. Cochran's Q test was used to compare the proportions of cases judged as hypo-response and hyper-response. If there was a statistically significant 
Table 1 Summary of baseline characteristics

\begin{tabular}{lc} 
Variable & Study cohort $(\mathrm{n}=43)$ \\
Age, years & $72.0(68.0-76.5)$ \\
Female sex & $6(14.0 \%)$ \\
Hypertension & $36(83.7 \%)$ \\
Diabetes mellitus & $17(39.5 \%)$ \\
Hyperlipidemia & $23(53.5 \%)$ \\
Coronary artery disease & $2(4.7 \%)$ \\
Current smoking & $12(27.9 \%)$ \\
Statin & $34(79.1 \%)$ \\
Proton pump inhibitor & $36(83.7 \%)$ \\
Cilostazol & $2(4.7 \%)$ \\
Oral anti-coagulant & $3(7.0 \%)$ \\
Duration of clopidogrel use before treatment, days & $27(18.5-85.5)$ \\
Hemoglobin, g/dL & $13.9(12.6-14.5)$ \\
Platelets, $\times 10^{3} / m L$ & $21.2(18.6-25.6)$ \\
Serum albumin, mg/dL & $4.2(3.9-4.4)$ \\
Symptomatic lesion & $21(48.8 \%)$ \\
NASCET, \%* & $69.3(62.5-79.9)$ \\
General anesthesia & $42(97.7 \%)$ \\
9-Fr guiding catheter & $36(83.7 \%)$ \\
Closed stent & $35(81.4 \%)$ \\
Flow reversal & $35(81.4 \%)$ \\
\hline Continuous variables are presented as median (interquartile range). * The degree of carotid \\
artery stenosis measured using the NASCET method. NASCET: North American Symptom- \\
atic Carotid Endarterectomy Trial & \\
&
\end{tabular}

difference between any of the measuring time points, the difference between the preprocedural value and the value at the measuring time point (delta PRU) was determined, and the factors affecting the difference were analyzed using multiple regression analysis. Statistical analysis was performed using R 3.3.3 GUI 1.69 Mavericks build (7328), and $\mathrm{p}<0.05$ was defined to be significantly different.

\section{Results}

The patient characteristics are summarized in Table 1. The median age of the 43 enrolled patients was 72.0 years (interquartile range: 68.0-76.5 years) including 37 males $(86.0 \%)$ and 6 females $(14.0 \%)$. Of the 43 patients, 21 (48.8\%) presented initially with ischemic events ipsilateral to the ICA stenosis. The median stenosis degree was 69.3 (62.5-79.7) according to the NASCET method. All procedures were performed under DAPT consisting of $100 \mathrm{mg} / \mathrm{d}$ aspirin and $75 \mathrm{mg} / \mathrm{d}$ clopidogrel. In two cases (4.7\%), $200 \mathrm{mg} / \mathrm{d}$ cilostazol was also administered. Oral anticoagulants in addition to DAPT were administered in three patients $(7.0 \%)$. The median duration of oral clopidogrel administration before the stenting procedure was $27 \mathrm{~d}$ (interquartile range: $18.5-85.5 \mathrm{~d}$ ).
In 42 patients (97.7\%), CAS was performed under general anesthesia, and in one patient (2.3\%) under local anesthesia. A 9-Fr guiding catheter was used in 36 patients $(83.7 \%)$, whereas in the remaining seven patients $(16.3 \%)$, either a 6-Fr ultra-long sheath or an 8-Fr guiding catheter was used. In $37(86.0 \%)$ and $6(14.0 \%)$ patients, CAS was performed via the femoral and right radial artery, respectively. Regarding emboli protection devices, a flow reversal system was used in 35 patients $(81.4 \%)$, distal protection in seven patients $(16.3 \%)$, and proximal protection in one patient $(2.3 \%)$. Closed- and open-cell stents were used in $35(81.4 \%)$ and $8(18.6 \%)$ patients, respectively.

Periprocedural complications were recorded in three cases $(7.0 \%)$. In one patient, it was a transient ischemic attack, and two patients presented cerebral infarction. One of them had improved symptoms at discharge; the other patient's neurological deficits became permanent. In 14 patients $(32.6 \%)$, high signal intensity spots appeared in DWI taken the day after the CAS procedure. There were no hemorrhagic complications. The overall morbidity and mortality rates were $2.3 \%$ and $0 \%$, respectively.

According to preprocedural ARU and PRU values determined by VerifyNow, seven patients $(16.3 \%)$ were categorized as hypo-response to aspirin, six patients $(14.0 \%)$ as 
Table 2 Comparison of the patient characteristics between aspirin reaction unit hypo-response and normal response

\begin{tabular}{lccc} 
Variables & Hypo-response & Normal response & p value \\
Age, years & $\mathrm{n}=7$ & $\mathrm{n}=36$ & 0.97 \\
Female sex & $1(69.5-77)$ & $72(68.0-77.0)$ & 1 \\
Hypertension & $7(100 \%)$ & $5(13.9 \%)$ & 0.58 \\
Diabetes mellitus & $5(71.4 \%)$ & $29(80.6 \%)$ & 0.09 \\
Hyperlipidemia & $4(57.1 \%)$ & $12(33.3 \%)$ & 1 \\
Coronary artery disease & $0(0 \%)$ & $19(52.8 \%)$ & 1 \\
Current smoking & $0(0 \%)$ & $2(5.6 \%)$ & 0.16 \\
Statin & $6(85.7 \%)$ & $12(33.3 \%)$ & 1 \\
Proton pump inhibitor & $7(100 \%)$ & $28(77.8 \%)$ & 0.58 \\
Cilostazol & $2(28.6 \%)$ & $29(80.6 \%)$ & $0.02 \dagger$ \\
Oral anti-coagulant & $2(28.6 \%)$ & $0(0 \%)$ & 0.06 \\
Hemoglobin, g/dL & $14.1(12.4-14.2)$ & $1(2.8 \%)$ & 0.55 \\
Platelets, $\times 10^{3 / m L}$ & $21.7(18.5-22.9)$ & $13.5(12.6-14.6)$ & 0.61 \\
Serum albumin, mg/dL & $4.2(3.7-4.4)$ & $21.2(18.7-26.3)$ & 0.43 \\
Preprocedural PRU & $160(94.0-219.5)$ & $4.3(3.9-4.4)$ & 0.52 \\
Symptomatic lesion & $5(71.4 \%)$ & $177(118.8-209.3)$ & 0.24 \\
NASCET, \%* & $80.2(71.0-86.2)$ & $16(44.4 \%)$ & $0.047 \neq$ \\
\hline
\end{tabular}

Continuous variables are presented as median (interquartile range). * The degree of carotid artery stenosis measured using the NASCET method. † Statistically significant in Fisher's exact test. ‡ Statistically significant in the Mann-Whitney U test. NASCET: North American Symptomatic Carotid Endarterectomy Trial; PRU: P2Y12 reaction unit

hypo-response to clopidogrel, and four patients $(9.3 \%)$ as hyper-response to clopidogrel. Thus, 33 patients $(76.7 \%)$ were within the therapeutic PRU range. Characteristics comparison between hypo-response and normal or hyper-response patients to aspirin and clopidogrel are shown in Tables 2 and 3. In the group with ARU hyporesponse, co-administration of cilostazol was more frequent $(28.6 \%$ vs. $0 \%, \mathrm{p}=0.023)$, and the NASCET degree of stenosis was higher $(80.2 \%$ [71.0-86.2\%] vs. $66.3 \%$ $[59.5-78.0 \%], \mathrm{p}=0.047)$. In the PRU hypo-response group, the levels of hemoglobin $(10.5 \mathrm{~g} / \mathrm{dL}[10.1-11.0 \mathrm{~g} /$ $\mathrm{dL}]$ vs. $14.1 \mathrm{~g} / \mathrm{dL}[13.1-14.5 \mathrm{~g} / \mathrm{dL}], \mathrm{p}=0.01)$ and serum albumin $(3.4 \mathrm{mg} / \mathrm{dL}[2.8-3.6 \mathrm{mg} / \mathrm{dL}]$ vs. $4.3 \mathrm{mg} / \mathrm{dL}$ [4.1-4.4 mg/dL], p <0.001) were significantly decreased.

Individual changes in ARU and PRU values are shown in Fig 1A and 1B. The median preprocedural ARU value was 408 (392-497), and the median post-procedural ARUs were $418(405.0-470.0)$ on day 1, $405(393.0-460.5)$ on day 3, and 402 (388.5-477.5) on day 7. The median ARU values were below the cutoff at all measured time points, and the multiple comparison test (MCT) did not reveal any statistically significant differences among these values. The percentages of patients classified as hyporesponse were $16.3 \%$ before CAS, $7.0 \%$ on day $1,2.3 \%$ on day 3 , and $7.0 \%$ on day 7 with no significant group differences according to Cochran's $Q$ test $(p=0.11)$. The median preprocedural PRU value was 173 (116.5-209.5) compared to median post-procedural PRUs of 233 (166.5273.5) on day 1, 139 (70.5-205.5) on day 3, and 51 (9.0-79.5) on day 7. The median PRU ranged between the upper and lower cutoff values before the procedure and on day 3; however, on day 1, the median PRU exceeded the upper cutoff, whereas it was below the lower cutoff value on day 7. The MCT revealed significant differences between day 0 and day 1 , as well as day 0 and day 7 . The percentages of cases categorized as hypo-response were significantly different ( $\mathrm{p}<0.001$, Cochran's $\mathrm{Q}$ test) with $14.0 \%$ before CAS, $51.2 \%$ on day $1,18.6 \%$ on day 3 , and $11.6 \%$ on day 7 . Similar significant differences $(p<0.001)$ were revealed for the percentages of hyper-responses with $9.3 \%$ before CAS, $2.3 \%$ on day $1,23.3 \%$ on day 3 , and $62.8 \%$ on day 7 . 
Table 3 Comparison of the patient characteristics between P2Y12 reaction unit hypo-response and normal or hyper-response

\begin{tabular}{|c|c|c|c|}
\hline Variables & $\begin{array}{l}\text { Hypo-response } \\
\qquad n=6\end{array}$ & $\begin{array}{l}\text { Normal or hyper-response } \\
\qquad n=37\end{array}$ & $p$ value \\
\hline Age, years & $72(71-76)$ & $72(68-76)$ & 0.69 \\
\hline Female sex & $2(33.3 \%)$ & $4(10.8 \%)$ & 0.19 \\
\hline Hypertension & $4(66.7 \%)$ & $32(86.5 \%)$ & 0.25 \\
\hline Diabetes mellitus & $3(50.0 \%)$ & $14(37.8 \%)$ & 0.67 \\
\hline Hyperlipidemia & $4(66.7 \%)$ & $19(51.4 \%)$ & 0.67 \\
\hline Coronary artery disease & $0(0 \%)$ & $2(5.4 \%)$ & 1 \\
\hline Current smoking & $1(16.7 \%)$ & $11(29.7 \%)$ & 0.66 \\
\hline Statin & $4(66.7 \%)$ & $30(81.1 \%)$ & 0.59 \\
\hline Proton pump inhibitor & $5(83.3 \%)$ & $31(83.8 \%)$ & 1 \\
\hline Cilostazol & $0(0 \%)$ & $2(5.4 \%)$ & 1 \\
\hline Oral anti-coagulant & $0(0 \%)$ & $3(8.1 \%)$ & 1 \\
\hline Duration of clopidogrel use before treatment, days & $22(18.0-26.8)$ & $41(20.0-88.0)$ & 0.25 \\
\hline Hemoglobin, g/dL & $10.5(10.1-11.0)$ & $14.1(13.1-14.5)$ & $0.01 \dagger$ \\
\hline Platelets, $\times 10^{3} / \mathrm{mL}$ & $17.4(15.0-25.5)$ & $21.5(19.1-25.3)$ & 0.20 \\
\hline Serum albumin, $\mathrm{mg} / \mathrm{dL}$ & $3.4(2.8-3.6)$ & $4.3(4.1-4.4)$ & $<0.001 \dagger$ \\
\hline Preprocedural ARU & $422.5(411.5-520.5)$ & $406.0(388.0-494.0)$ & 0.10 \\
\hline Symptomatic lesion & $5(83.3 \%)$ & $16(43.2 \%)$ & 0.09 \\
\hline NASCET, \%* & $74.6(66.1-88.4)$ & $69.3(59.6-78.9)$ & 0.21 \\
\hline
\end{tabular}

Continuous variables are presented as median (interquartile range). ${ }^{*}$ The degree of carotid artery stenosis measured using the NASCET method. †Statistically significant in the Mann-Whitney U test. ARU: aspirin reaction unit; NASCET: North American Symptomatic Carotid Endarterectomy Trial

There was neither statistically significant relation between ARU nor PRU responsiveness at each measured time point and clinical adverse events (Table 4).

Next, we performed multiple regression analyses to presume the factors affecting individual PRU changes between the value before CAS and the value on day 1 or day 7 , respectively. Due to the small case number, the purpose of this analysis was merely to consider the possibility. The results of the multiple regression analysis are shown in Tables 5 and $\mathbf{6}$. Regarding the differences between PRU values before the CAS and on day 1, the preprocedural ARU value, the NASCET degree of stenosis, and serum albumin level were extracted as possible factors. On the other hand, none was extracted as a possible factor of the difference between PRU values prior to CAS and on day 7 .

\section{Discussion}

To balance the risk between hemorrhagic and ischemic complications, interventional procedures recommend monitoring the platelet function and adjusting the dosages of antiplatelet agents. The high level of variability in antiplatelet response to aspirin and clopidogrel among patients is well recognized. However, there are few reports about individual changes in antiplatelet response during neurointerventional procedures. In this CAS study, individual ARU values were stable over time; however, the PRU values varied in the periprocedural period. The median PRU values on day 1 and day 7 after CAS were outside the defined normal range. Although $76.7 \%$ of patients were before CAS within the therapeutic clopidogrel range, $51.2 \%$ of cases were categorized as hypo-response on day 1 after CAS, whereas $62.9 \%$ of cases were classified as hyper-response on day 7 . We successfully placed the stent in all enrolled patients, and a permanent periprocedural complication occurred in only one patient $(2.3 \%)$. The results of the JR-NET3, a nationwide survey in Japan, showed that the incidence of clinically significant complications is $2.3 \%{ }^{25)}$ Therefore, our clinical treatment results are acceptable, and the data on antiplatelet agents can be used for further analyses. 

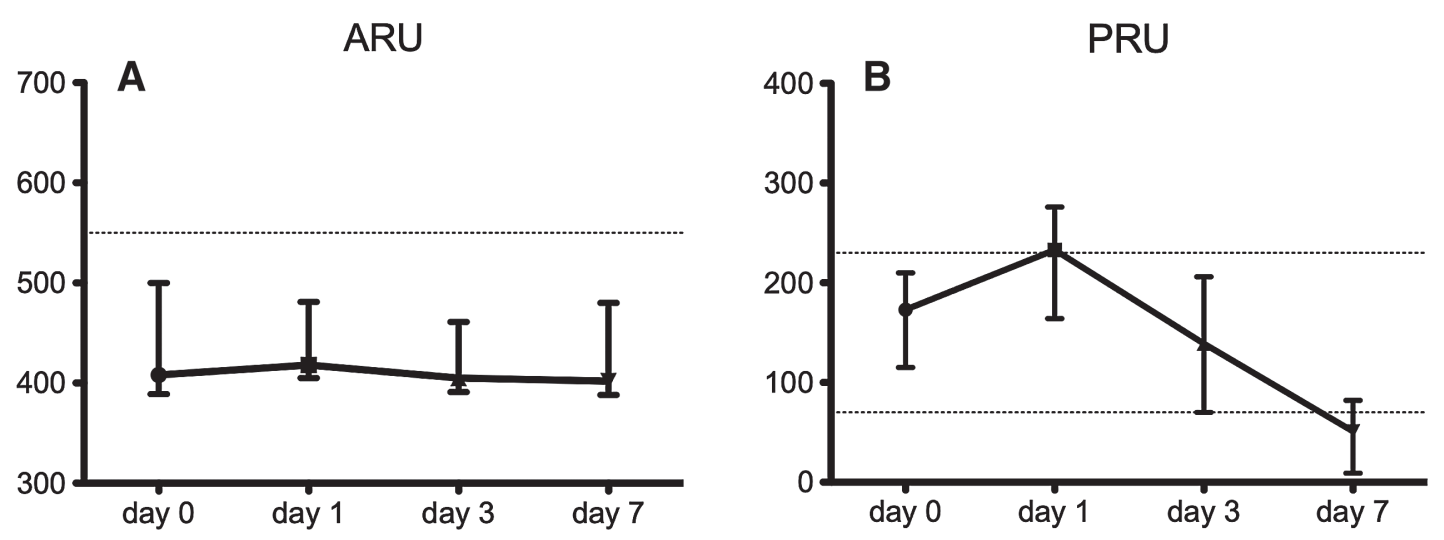

Fig. 1 Time-dependent changes in ARU and PRU values. (A) ARU changes. The dotted line indicates the cutoff value. No significant differences between any of these values were detected (Wilcoxon signed-rank test adjusted by Holm's method). (B) Changes in PRU values. The dotted lines indicate upper and lower cutoff values. All combinations of time points differ significantly (Wilcoxon signed-rank test adjusted by Holm's method). ARU: aspirin reaction unit; PRU: P2Y12 reaction unit

\section{Factors causing preprocedural hypo-response to antiplatelet drugs}

Various predictors for hypo-response to aspirin such as increased body weight, body mass index, and proteinuria have been reported. ${ }^{6,7)}$ In the present study, co-administration of cilostazol and a higher NASCET degree of stenosis were more frequent in the ARU hypo-response group. A double-blind randomized multi-center trial in Korea revealed that cilostazol add-on to aspirin in ischemic stroke patients made no significant difference in the percentage of aspirin resistance compared to placebo ${ }^{8)}$; however, the mean ARU value before treatment had the tendency to be higher in the cilostazol group. Since the co-administration of cilostazol was in our study only observed in two patients, further investigations are warranted. The literature does not describe a correlation between the degree of carotid artery stenosis and ARU values. In carotid artery stenosis, aspirin is administered to prevent the progress of the stenosis. The ARU values are likely to be increased in patients with severe stenosis because the stenosis in some aspirin hypo-responders may progress to a CAS indication.

Numerous factors such as age, sex, hypertension, diabetes mellitus, obesity, and CYP2C19 polymorphism have been reported as predictors for hypo-response to clopidogrel. ${ }^{14,15)}$ Current smoking, as well as levels of hemoglobin, cholinesterase, and serum albumin, have been also reported to be correlated with hypo-response to clopidogrel. ${ }^{9,16,17)}$ In the present study, age, sex, hypertension, diabetes mellitus, and smoking did not correlate with hypo-response to clopidogrel; however, the levels of hemoglobin and serum albumin were significantly lower in the PRU hypo-response group.

\section{Individual ARU and PRU changes and their predictors}

Multiple repeat measurements of the VerifyNow assay in healthy volunteers who did not take antiplatelet drugs revealed that PRU values varied widely intraindividually in contrast to ARU values. ${ }^{26)}$ There are some reports regarding the variability of ARU and PRU values during periprocedural periods of endovascular treatments, ${ }^{10,18,21-23)}$ but most investigated the relatively long-term variability such as more than 1 week to 6 months after treatment. ${ }^{10,18,21)}$ Khanna et al. measured ARU and PRU values in patients after percutaneous coronary intervention (PCI) at discharge and 1 week, 1 month, 3 months, and 6 months after discharge. They describe that the ARU was unchanged for the study period but the PRU was significantly increased at 1 month after discharge with no significant changes afterward. ${ }^{10)}$ Tello-Montoliu et al. compared the PRU values of patients undergoing PCI at discharge, 3 months, and 6 months. These authors report that the PRU tended to be higher after 3 months than at discharge, and there was no difference between 3 months and 6 months. ${ }^{21)}$ Watanabe et al. compared PRUs at $7 \mathrm{~d}$ and $30 \mathrm{~d}$ after transcatheter aortic valve implantation and reported that the PRUs were not significantly different $(136.7 \pm 73.4$ and $150.4 \pm 83.2$, respectively, $\mathrm{p}=0.13){ }^{18)}$ Patients included in their study had taken aspirin $100 \mathrm{mg} / \mathrm{d}$ for $7 \mathrm{~d}$ or more and either clopidogrel 75 $\mathrm{mg} / \mathrm{d}$ for $7 \mathrm{~d}$ or more or alternatively loading with $300 \mathrm{mg}$ followed by $75 \mathrm{mg} / \mathrm{d}$ before the treatment. These reports indicate that the ARU is stable over time, whereas the PRU stabilizes after 1 week or more after the procedure.

Regarding the short-term variability in PRU values, they were in another study significantly decreased $4 \mathrm{~h}$ after 


\begin{tabular}{|c|c|c|c|c|c|c|}
\hline \multirow[b]{2}{*}{ Variables } & \multicolumn{3}{|c|}{ Symptomatic complication } & \multicolumn{3}{|c|}{ Appearance of DWI-positive lesion } \\
\hline & $\begin{array}{c}(+) \\
(n=3)\end{array}$ & $\begin{array}{c}(-) \\
(n=40)\end{array}$ & $p$ & $\begin{array}{c}(+) \\
(n=14)\end{array}$ & $\begin{array}{c}(-) \\
(n=29)\end{array}$ & $p$ \\
\hline \multicolumn{7}{|c|}{ ARU hypo-response } \\
\hline Preprocedural & $0(0 \%)$ & $7(17.5 \%)$ & 1 & $2(14.3 \%)$ & $5(17.2 \%)$ & 1 \\
\hline On day1 & $0(0 \%)$ & $3(7.5 \%)$ & 1 & $0(0 \%)$ & $3(10.3 \%)$ & 1 \\
\hline On day3 & $0(0 \%)$ & $1(2.5 \%)$ & 1 & $1(7.1 \%)$ & $0(0 \%)$ & 0.33 \\
\hline On day 7 & $0(0 \%)$ & $3(7.5 \%)$ & 1 & $0(0 \%)$ & $3(10.3 \%)$ & 0.54 \\
\hline \multicolumn{7}{|c|}{ PRU hypo-response } \\
\hline Preprocedural & $0(0 \%)$ & $6(15.0 \%)$ & 1 & $1(7.1 \%)$ & $5(17.2 \%)$ & 0.65 \\
\hline On day 1 & $2(66.7 \%)$ & $20(50.0 \%)$ & 1 & $7(50.0 \%)$ & $15(51.7 \%)$ & 1 \\
\hline On day3 & $1(33.3 \%)$ & $7(17.5 \%)$ & 0.47 & $4(28.6 \%)$ & $4(13.8 \%)$ & 0.40 \\
\hline On day7 & $1(33.3 \%)$ & $4(10.0 \%)$ & 0.32 & $2(14.3 \%)$ & 3 (10.3\%) & 1 \\
\hline \multicolumn{7}{|c|}{ PRU hyper-response } \\
\hline Preprocedural & $0(0 \%)$ & $4(10.0 \%)$ & 1 & $2(14.3 \%)$ & $2(6.9 \%)$ & 0.59 \\
\hline On day1 & $0(0 \%)$ & $1(2.5 \%)$ & 1 & $1(7.1 \%)$ & $0(0 \%)$ & 0.33 \\
\hline On day3 & $0(0 \%)$ & $10(25.0 \%)$ & 1 & $3(21.4 \%)$ & 7 (24.1\%) & 1 \\
\hline On day 7 & $2(66.7 \%)$ & $25(62.5 \%)$ & 1 & $9(64.3 \%)$ & $18(62.1 \%)$ & 1 \\
\hline
\end{tabular}

$A R U$ : aspirin reaction unit; DWI: diffusion-weighed image; PRU: $P 2 Y 12$ reaction unit

PCI compared to values before PCI, and there was no significant difference between 4 and $24 \mathrm{~h}$ after PCI. ${ }^{22)}$ The difference between these and our results may be explained by the presence or absence of premedication. In their study, the premedication was a loading dose of $300 \mathrm{mg}$ clopidogrel just before the PCI, whereas in our study, CAS was performed after a sufficiently long premedication period of more than 1 week for aspirin and 2 weeks for clopidogrel. The findings by Lee et al. regarding stenting for cerebral artery stenosis are similar to our results. ${ }^{23)}$ In their study, the median ARU increased after stenting from 418 (range: 350-586) to 469 (range: $389-573 ; \mathrm{p}=0.045$ ) and the median PRU from 256 (range: 56-325) to 274 (range: 81-370; $\mathrm{p}=0.018$ ). Their patients had taken $100 \mathrm{mg} / \mathrm{d}$ aspirin and $75 \mathrm{mg} / \mathrm{d}$ clopidogrel for $5 \mathrm{~d}$ or a loading dose of 300-500 mg aspirin and $300 \mathrm{mg}$ clopidogrel before the stenting procedure.

One of the reasons why PRU values are elevated $24 \mathrm{~h}$ after a procedure might be that stenting procedures are invasive to blood vessel walls. In our study, an arterial line was inserted, and a relatively large-diameter catheter was used for stent delivery and distal embolic protection in almost all patients. Besides, CAS is a procedure that presses metal into the vessel wall and involves dilation of the vessel by a balloon. Muller et al. suggest increased platelet reactivity in the presence of impaired endothelial functions. ${ }^{27)}$ The CAS-induced damage to the endothelium may activate platelets, which may explain why the PRU was elevated on day 1 . Schofer et al. reported that DWIpositive lesions increased over time after CAS. ${ }^{28)}$ MRIs were taken twice at a mean of $3.5 \mathrm{~h}$ and $18 \mathrm{~h}$ after CAS, and DWI-positive lesions appeared in $20 \%$ and $32 \%$ of the patients, respectively. Moreover, in $5 \%$ of the cases, the number of DWI-positive lesions were increased in the second image. Among 111 patients undergoing CAS in the publication by Qureshi et al., perioperative ischemic complications occurred in 14 patients - in 4 patients during the procedure and in 10 patients within $48 \mathrm{~h}$ after the procedure. ${ }^{29)}$ Both reports demonstrate that CAS-induced thromboembolic complications appear after rather than during the procedure, which may be explained by PRU increases on day 1 . The values on day 1 might be affected by differences in perioperative management such as perioperative infusions, bleedings from puncture site and connector, or blood samples drawn from the arterial line. However, there were no significant differences between the ARU value on day 1 and those at other time points. We suggest that the perioperative management had little effect on PRU values. 
Table 5 Multiple regression analysis for differences between PRU on day 1 and preprocedural PRU

\begin{tabular}{|c|c|c|c|c|c|c|c|c|}
\hline & $\begin{array}{c}\text { Regression } \\
\text { coefficient estimate }\end{array}$ & Standard error & Lower $95 \% \mathrm{Cl}$ & Upper 95\% Cl & $\begin{array}{l}\text { Standardized regression } \\
\text { coefficient estimate }\end{array}$ & t-value & $p$ value & VIF \\
\hline (Intercept) & -351.252 & 148.955 & -656.881 & -45.622 & & -2.358 & 0.026 & $\dagger$ \\
\hline Age & 0.434 & 1.081 & -1.783 & 2.652 & 0.060 & 0.402 & 0.691 & 1.373 \\
\hline Female & 29.762 & 17.504 & -6.152 & 65.677 & 0.248 & 1.700 & 0.101 & 1.299 \\
\hline Hypertension & -19.617 & 18.632 & -57.847 & 18.613 & -0.174 & -1.053 & 0.302 & 1.671 \\
\hline Diabetes mellitus & -1.188 & 13.202 & -28.276 & 25.899 & -0.014 & -0.090 & 0.929 & 1.471 \\
\hline Hyperlipidemia & 10.851 & 13.464 & -16.774 & 38.477 & 0.130 & 0.806 & 0.427 & 1.593 \\
\hline Current smoking & -5.757 & 13.964 & -34.408 & 22.894 & -0.062 & -0.412 & 0.683 & 1.385 \\
\hline Statin & -16.355 & 16.064 & -49.316 & 16.606 & -0.160 & -1.018 & 0.318 & 1.508 \\
\hline Proton pump inhibitor & -19.068 & 19.216 & -58.496 & 20.361 & -0.169 & -0.992 & 0.330 & 1.777 \\
\hline Hemoglobin & 5.744 & 4.978 & -4.470 & 15.958 & 0.228 & 1.154 & 0.259 & 2.388 \\
\hline Platelets & 0.367 & 1.128 & -1.947 & 2.681 & 0.057 & 0.325 & 0.747 & 1.846 \\
\hline Serum albumin & 35.094 & 16.724 & 0.779 & 69.409 & 0.446 & 2.098 & 0.045 & $2.755 \dagger$ \\
\hline Preprocedural ARU & 0.253 & 0.090 & 0.068 & 0.437 & 0.445 & 2.808 & 0.009 & $1.533+$ \\
\hline Preprocedural PRU & 0.069 & 0.110 & -0.156 & 0.294 & 0.115 & 0.627 & 0.536 & 2.064 \\
\hline Symptomatic lesion & -16.551 & 12.546 & -42.292 & 9.191 & -0.199 & -1.319 & 0.198 & 1.389 \\
\hline NASCET* & 0.956 & 0.437 & 0.058 & 1.853 & 0.336 & 2.185 & 0.038 & $1.440 \dagger$ \\
\hline
\end{tabular}

${ }^{*}$ The degree of carotid artery stenosis measured using the NASCET method. †Statistically significant. ARU: aspirin reaction unit; Cl: confidence interval; NASCET: North American Symptomatic Carotid Endarterectomy Trial; PRU: P2Y12 reaction unit 
Table 6 Multiple regression analysis for differences between PRU on day 7 and preprocedural PRU

\begin{tabular}{|c|c|c|c|c|c|c|c|c|}
\hline & $\begin{array}{c}\text { Regression } \\
\text { coefficient estimate }\end{array}$ & Standard error & Lower $95 \% \mathrm{Cl}$ & Upper 95\% Cl & $\begin{array}{l}\text { Standardized regression } \\
\text { coefficient estimate }\end{array}$ & $\mathrm{t}$-value & $p$ value & VIF \\
\hline (Intercept) & 192.274 & 335.954 & -497.047 & 881.593 & & 0.572 & 0.572 & \\
\hline Age & -1.069 & 2.438 & -6.071 & 3.932 & -0.079 & -0.439 & 0.664 & 1.373 \\
\hline Female & -3.897 & 39.478 & -84.899 & 77.106 & -0.017 & -0.099 & 0.922 & 1.299 \\
\hline Hypertension & -41.776 & 42.023 & -128.000 & 44.449 & -0.197 & -0.994 & 0.329 & 1.671 \\
\hline Diabetes mellitus & 8.635 & 29.775 & -52.458 & 69.728 & 0.054 & 0.290 & 0.774 & 1.471 \\
\hline Hyperlipidemia & -10.858 & 30.367 & -73.165 & 51.449 & -0.069 & -0.358 & 0.723 & 1.593 \\
\hline Current smoking & -26.379 & 31.494 & -90.998 & 38.240 & -0.151 & -0.838 & 0.410 & 1.385 \\
\hline Statin & 33.497 & 36.231 & -40.844 & 107.837 & 0.174 & 0.925 & 0.363 & 1.508 \\
\hline Proton pump inhibitor & -26.924 & 43.341 & -115.851 & 62.004 & -0.127 & -0.621 & 0.540 & 1.777 \\
\hline Hemoglobin & -11.703 & 11.227 & -34.740 & 11.334 & -0.247 & -1.042 & 0.307 & 2.388 \\
\hline Platelets & -1.337 & 2.543 & -6.555 & 3.882 & -0.109 & -0.526 & 0.604 & 1.846 \\
\hline Serum albumin & -4.955 & 37.720 & -82.349 & 72.440 & -0.033 & -0.131 & 0.897 & 2.755 \\
\hline Preprocedural ARU & 0.065 & 0.203 & -0.351 & 0.481 & 0.061 & 0.320 & 0.751 & 1.533 \\
\hline Preprocedural PRU & -0.496 & 0.247 & -1.003 & 0.011 & -0.441 & -2.006 & 0.055 & 2.064 \\
\hline Symptomatic lesion & -46.057 & 28.295 & -104.114 & 12.001 & -0.294 & -1.628 & 0.115 & 1.389 \\
\hline NASCET* & 1.642 & 0.987 & -0.382 & 3.666 & 0.306 & 1.665 & 0.108 & 1.440 \\
\hline
\end{tabular}

${ }^{*}$ The degree of carotid artery stenosis measured using the NASCET method. There is no statistically significant difference. ARU: aspirin reaction unit; Cl: confidence interval; NASCET: North American Symptomatic Carotid Endarterectomy Trial; PRU: P2Y12 reaction unit 
As possible factors affecting PRU increases on day 1, the preprocedural ARU value, NASCET degree of stenosis, and serum albumin level were extracted. Buch et al. and Lev et al. found a positive correlation between ARU and PRU levels. ${ }^{11,30)}$ The mechanism of this correlation is unknown; however, patients with poor aspirin response may have reduced responsiveness to clopidogrel. The NASCET degree of stenosis was in the current study correlated with the preprocedural ARU value, which might be the reason why the NASCET degree of stenosis was also correlated with increases in PRU. Serum albumin has been reported as a predictor for hyporesponse to clopidogrel, ${ }^{9,17)}$ and in our results, albumin was significantly lower in the preprocedural hypo-response group. This low serum albumin level may reflect a decrease in liver functions, and various drugs used in general anesthesia may have further inhibited the clopidogrel metabolism.

The ARU values did not change on day 3 and day 7 , whereas PRU values decreased significantly from day 1 to day 3 and from day 3 to day 7. Moreover, the PRU values on day 7 were significantly different from preprocedural values. Several reports have already pointed out that PRU values are decreased after treatment. ${ }^{19,20)}$ Delgado Almandoz et al. reported a delayed conversion into hyperresponse to clopidogrel. ${ }^{19)}$ In their study, 36 patients who underwent endovascular treatment for unruptured cerebral aneurysm consistently received $75 \mathrm{mg} / \mathrm{d}$ clopidogrel during the study period and received repeated VerifyNow assay on the day before treatment and 30-40 d after initiation of clopidogrel administration. In these patients, mean duration of administration before first test and follow-up test were $8.8 \mathrm{~d}$ and $30.2 \mathrm{~d}$, respectively. Two of 36 patients were considered hyper-response and 34 patients were within the therapeutic range (60-240) in the first test; however, 20 of these 34 patients $(58.8 \%)$ converted to hyper-response in follow-up test. Kayan et al. verify the efficacy of a two-test protocol for achieving therapeutic response to clopidogrel prior to endovascular intracranial aneurysm treatment. ${ }^{20)}$ Patients received clopidogrel $17 \mathrm{~d}$ before treatment, and PRU was measured twice before the procedure. In the first and second tests, $71.8 \%$ and $89.3 \%$ of the patients, respectively, were within the range of the test but 51 of the 82 enrolled patients $(62.2 \%)$ converted into hyper-responders in the postoperative test. According to these results, the responsiveness to clopidogrel may change depending on the duration of clopidogrel administration. In the current study, the duration of oral clopidogrel administration was at least $14 \mathrm{~d}$, and the median duration from clopidogrel initiation to the first VerifyNow test was $27 \mathrm{~d}$. The duration of the premedication was sufficient; however, the PRU value on day 7 was significantly decreased compared to the preprocedural value, and more than half of the patients were hyper-responsive to clopidogrel. This delayed conversion to clopidogrel hyper-response may not have been caused by the duration of clopidogrel use but by the intervention itself. In our study, none was extracted as a possible factor affecting PRU decreases on day 7. Kayan et al. who were mentioned above performed a multiple logistic regression analysis to identify predictors of delayed conversion into hyper-response in their study; however, none was found. ${ }^{20)}$ They hypothesized that postoperative hyper-response might be induced, possibly related to a postoperative change in hepatic clopidogrel metabolism related to general anesthesia or the physiological stress of the procedure.

In this study, only one permanent symptomatic ischemic complication and no hemorrhagic complication occurred and no correlation was found between complications and PRU values; however, various authors have reported that higher PRU values correlated with ischemic complications and lower PRU values with hemorrhagic complications. ${ }^{4,13,18,19)}$ From our results, PRU values are likely to decrease after stenting procedures. Thus, we need to be careful not to induce hemorrhagic infarctions. PRU evaluations should be timely to allow appropriate adjustments of antiplatelet drugs. Since there were no significant ARU changes, no further monitoring would be needed for cases showing normal responses before the CAS procedure.

\section{Limitations}

There are several limitations to this study. First, it had a retrospective design, and the sample size was small. Second, one patient $(2.3 \%)$ had permanent ischemic sequelae, and 14 patients $(32.6 \%)$ presented high signal intensity spots in DWIs taken on the day after CAS. Due to the small sample size, correlations with PRU values could not be determined. Further studies are needed to reveal whether individual PRU values are correlated with clinical outcomes or not. Third, we do not survey CYP2C19 polymorphisms, a strong predictor of clopidogrel hypo-response. ${ }^{15)}$ Additional studies are required to examine correlations between CYP2C19 polymorphisms and time-dependent PRU variability.

\section{Conclusion}

This study evaluated periprocedural platelet functions using VerifyNow in patients undergoing CAS. The individual 
ARU values were stable throughout the entire measurement period. By contrast, PRU values varied substantially; they exceeded the upper cutoff value on the day following the CAS procedure but were decreased below the lower cutoff value on the seventh postprocedural day. PRU evaluations should be timely to allow appropriate adjustments of antiplatelet drugs. Since there were no significant ARU changes, no further monitoring would be needed for cases showing normal responses before the CAS procedure.

\section{Acknowledgment}

The authors thank Dr. Masako Akiyama, Ph.D., Medical Innovation Promotion Center, Tokyo Medical and Dental University for her advice on the statistical analyses. This research received no specific grant from any funding agency in the public, commercial, or not-for-profit sectors. We would like to thank Editage (www.editage.com) for English language editing.

\section{Disclosure Statement}

The authors report no conflicts of interest concerning the materials or methods used in this study or the findings specified in this paper.

\section{References}

1) Yadav JS, Wholey MH, Kuntz RE, et al: Protected carotid-artery stenting versus endarterectomy in high-risk patients. N Engl J Med 2004; 351: 1493-1501.

2) Jongen LM, Hendrikse J, Waaijer A, et al: Frequency and consequences of early in-stent lesions after carotid artery stent placement. J Vasc Interv Radiol 2009; 20: 573-579.

3) Chaturvedi S, Yadav JS: The role of antiplatelet therapy in carotid stenting for ischemic stroke prevention. Stroke 2006; 37: 1572-1577.

4) Kim KS, Fraser JF, Grupke S, et al: Management of antiplatelet therapy in patients undergoing neuroendovascular procedures. J Neurosurg 2018; 129: 890-905.

5) Price MJ, Berger PB, Teirstein PS, et al: Standard- vs highdose clopidogrel based on platelet function testing after percutaneous coronary intervention: the GRAVITAS randomized trial. JAMA 2011; 305: 1097-1105.

6) George G, Patel N, Jang C, et al: Proteinuria predicts resistance to antiplatelet therapy in ischemic stroke. Transl Stroke Res 2018; 9: 130-134.

7) Furtado RHM, Giugliano RP, Dalcoquio TF, et al: Increased bodyweight and inadequate response to aspirin in individuals with coronary artery disease. $J$ Thromb Thrombolysis 2019; 48: 217-224.
8) Lee JH, Cha JK, Lee SJ, et al: Addition of cilostazol reduces biological aspirin resistance in aspirin users with ischaemic stroke: a double-blind randomized clinical trial. Eur J Neurol 2010; 17: 434-442.

9) Su J, Li X, Yu Q, et al: Association of P2Y12 gene promoter DNA methylation with the risk of clopidogrel resistance in coronary artery disease patients. Biomed Res Int 2014; 2014: 450814.

10) Khanna V, Mikael R, Thayalasamy K, et al: Does the response to aspirin and clopidogrel vary over 6 months in patients with ischemic heart disease? J Thromb Haemost 2015; 13: 920-930.

11) Buch AN, Singh S, Roy P, et al: Measuring aspirin resistance, clopidogrel responsiveness, and postprocedural markers of myonecrosis in patients undergoing percutaneous coronary intervention. Am J Cardiol 2007; 99: 1518-1522.

12) Diener HC, Bogousslavsky J, Brass LM, et al: Aspirin and clopidogrel compared with clopidogrel alone after recent ischaemic stroke or transient ischaemic attack in high-risk patients (MATCH): randomised, double-blind, placebocontrolled trial. Lancet 2004; 364: 331-337.

13) Daou B, Starke RM, Chalouhi N, et al: P2Y12 reaction units: effect on hemorrhagic and thromboembolic complications in patients with cerebral aneurysms treated with the pipeline embolization device. Neurosurgery 2016; 78: 27-33.

14) Yaseen IF, Farhan HA, Abbas HM: Clopidogrel nonresponsiveness in patients undergoing percutaneous coronary intervention using the VerifyNow test: frequency and predictors. Eur J Hosp Pharm 2019; 26: 113-116.

15) Lin $\mathrm{M}$, Todaro $\mathrm{M}$, Chan J, et al: Association between CYP2C19 polymorphisms and outcomes in cerebral endovascular therapy. AJNR Am J Neuroradiol 2016; 37: 108-113.

16) Wadowski PP, Kopp CW, Koppensteiner R, et al: Decreased platelet inhibition by $\mathrm{P} 2 \mathrm{Y} 12$ receptor blockers in anaemia. Eur J Clin Invest 2018; 48. doi: 10.1111/eci.12861

17) Gremmel T, Mueller M, Koppensteiner R, et al: Liver function is associated with response to clopidogrel therapy in patients undergoing angioplasty and stenting. Angiology 2016; 67: 835-839.

18) Watanabe $Y$, Kozuma K, Ishikawa S, et al: Hyper-response to clopidogrel in Japanese patients undergoing transcatheter aortic valve implantation. Int Heart J 2016; 57: 190-197.

19) Delgado Almandoz JE, Kadkhodayan Y, Crandall BM, et al: Variability in initial response to standard clopidogrel therapy, delayed conversion to clopidogrel hyper-response, and associated thromboembolic and hemorrhagic complications in patients undergoing endovascular treatment of unruptured cerebral aneurysms. J Neurointerv Surg 2014; 6: 767-773.

20) Kayan Y, Delgado Almandoz JE, Fease JL, et al: Efficacy of a two-test protocol for achieving a therapeutic response to clopidogrel prior to elective endovascular intracranial 
aneurysm treatment and an 'induced' postoperative hyper-response. J Neurointerv Surg 2017; 9: 792-796.

21) Tello-Montoliu A, Rivera J, Hernández D, et al: Temporal changes in platelet response in acute coronary syndrome patients with prasugrel and clopidogrel after stent implantation. Circ J 2018; 82: 353-360.

22) Yang A, Pon Q, Lavoie A, et al: Long-term pharmacodynamic effects of Ticagrelor versus clopidogrel in fibrinolytictreated STEMI patients undergoing early PCI. J Thromb Thrombolysis 2018; 45: 225-233.

23) Lee DH, Kim HS, Kim SM, et al: Change of platelet reactivity to antiplatelet therapy after stenting procedure for cerebral artery stenosis: VerifyNow antiplatelet assay before and after stenting. Neurointervention 2012; 7: 23-26.

24) North American Symptomatic Carotid Endarterectomy Trial Collaborators, Barnett HJM, Taylor DW, et al: Beneficial effect of carotid endarterectomy in symptomatic patients with high-grade carotid stenosis. $N$ Engl J Med 1991; 325: 445-453.

25) Tokuda R, Yoshimura S, Uchida K, et al: Real-world experience of carotid artery stenting in Japan: analysis of 8458 cases from the JR-NET3 Nationwide Retrospective
Multi-center Registries. Neurol Med Chir (Tokyo) 2019; 59: 117-125.

26) Katzman BM, Wockenfus AM, Scott RJ, et al: Estimating short- and long-term reference change values and index of individuality for tests of platelet function. Clin Biochem 2019; 74: 54-59.

27) Muller O, Hamilos M, Bartunek J, et al: Relation of endothelial function to residual platelet reactivity after clopidogrel in patients with stable angina pectoris undergoing percutaneous coronary intervention. Am J Cardiol 2010; 105: 333-338.

28) Schofer J, Arendt M, Tübler T, et al: Late cerebral embolization after emboli-protected carotid artery stenting assessed by sequential diffusion-weighted magnetic resonance imaging. JACC Cardiovasc Interv 2008; 1: 571-577.

29) Qureshi AI, Luft AR, Janardhan V, et al: Identification of patients at risk for periprocedural neurological deficits associated with carotid angioplasty and stenting. Stroke 2000; 31: 376-382.

30) Lev EI, Patel RT, Maresh KJ, et al: Aspirin and clopidogrel drug response in patients undergoing percutaneous coronary intervention: the role of dual drug resistance. $\mathrm{J} \mathrm{Am}$ Coll Cardiol 2006; 47: 27-33. 